\title{
Trastornos musculoesqueléticos en los técnicos de laboratorio
}

Musculoskeletal Problems in Laboratory Technicians

\section{María Jesús López González}

${ }^{1}$ Universidad Internacional de La Rioja, Logroño, Spain

Fechas - Dates

Recibido: 2020.02.08

Publicado: 2020.04.15
Sección coordinada por / Section Coordinator

Dr. Guillermo García González

Profesor Agregado Derecho del Trabajo y SS Universidad Internacional de La Rioja (UNIR)

Email: guillermo.garcia@unir.net 


\section{Entrevista con María Jesús López González}

\section{1. ¿Cuál es la procedencia, especialización y áreas de trabajo de los autores del artículo? ${ }^{(1)}$ ¿Qué características le parecen más destacables de este grupo de trabajo?}

Las tres autoras del artículo, Ma Jesús López-González, Eva González-Menéndez y Silvia González, nos hemos formado en la Facultad de Química, en la Universidad de Oviedo, continuando nuestros estudios de Doctorado en el área de Ingeniería y Arquitectura, concretamente dentro del Programa de Ingeniería Química, Ambiental y Bioalimentaria con Mención hacia la Excelencia, en el departamento de Microbiología y Biotecnología de los alimentos (IPLA-CSIC). Tras más de 10 años de experiencia en el campo de la Prevención de Riesgos Laborales (PRL), en la actualidad somos profesoras en la Escuela Superior de Ingeniería y Tecnología (ESIT), de la Universidad Internacional de la Rioja (UNIR), dentro del Máster Universitario de PRL y del Máster en Sistemas Integrados de Gestión. En el ámbito investigador pertenecemos al grupo de investigación "Trabajo líquido y riesgos emergentes en la sociedad de la información, TR3S-i". Dentro del grupo, creamos la línea de investigación: "Riesgos emergentes derivados de la convergencia tecnológica y organizativa: riesgos químicos, ergonómicos y Biológicos" (RETO QERB). En el marco de esa línea, nos hemos especializado en el área de ergonomía y psicosociología aplicada, realizando varias publicaciones en revistas de impacto.

Inmersos en la cuarta revolución industrial, marcada por la velocidad de expansión en el uso de las TIC en el ámbito laboral, se hace totalmente necesario abordar el estudio de los riesgos emergentes cada vez más presentes en un entorno de trabajo líquido, marcado fundamentalmente por cambios organizativos en las formas de trabajo. En este contexto, el grupo de investigación TR3S-i está formado por un equipo multidisciplinar que permite realizar estudios, desde un enfoque holístico e integrador aportando resultados teóricos fundamentados científicamente que resultan transferibles a las organizaciones productivas, siendo a nuestro parecer lo más destacable de este grupo.

\section{2. ¿Cómo se ha financiado el estudio?}

Este estudio se ha financiado a través del Grupo de Investigación: "Trabajo líquido y riesgos emergentes en la sociedad de la información- TR3S-i, gracias al apoyo de la Universidad Internacional de la Rioja (UNIR), en el marco de su programa de investigación [2016-2018].

\section{3. ¿Qué problema pretende abordar este estudio y dónde radica su interés o relevancia para la seguridad y salud en el trabajo?}

Lo cierto es que el proyecto nace fruto de nuestra propia experiencia profesional, en el año 2014 aterrizamos en un laboratorio, para desarrollar la tesis doctoral, y fue con el paso de los meses, cuando observamos que empezábamos a sufrir dolencias musculares principalmente en cuello, hombro y muñeca, claramente asociadas a las actividades propias que se desarrollan en un laboratorio. Hablando con los doctorandos y los técnicos del laboratorio, comprobamos que compartían 
nuestra situación y lo relacionaban con tareas que conllevan el uso de equipos como la micropipeta, el microscopio o el uso del ordenador, entre otros. Esto nos hizo pensar que había una problemática generalizada.

Al buscar en la literatura científica observamos que había un vacío importante en la disponibilidad de estudios en población española centrados exclusivamente en este tipo de profesionales. De ahí nuestro interés por poner de manifiesto, con base científica, las partes del cuerpo más afectadas por dolencias musculoesqueléticas y establecer las variables personales y organizacionales que se asocian significativamente a esas molestias. Se detecta un porcentaje de técnicos afectados realmente elevado, estamos hablando de un $84.5 \%$, en un sector feminizado donde no se están llevando a cabo las medidas preventivas adecuadas de seguridad y salud.

\section{4. ¿Qué aporta este estudio de novedoso o destacable en relación al resto de producción científica sobre el problema estudiado?}

Las últimas estadísticas señalan los trastornos musculoesqueléticos como una de las problemáticas más acusadas que afecta a la población trabajadora en el mundo, lo que ha conllevado gran cantidad de investigaciones al respecto. Sin embargo, la mayoría de estudios en técnicos de laboratorio se centran en aspectos higiénicos y apenas existe literatura científica que aborde factores de riesgo ergonómico. En este sector, las medidas tomadas se apoyan básicamente en los estudios centrados en otras profesiones del ámbito sanitario.

Por otro lado, hemos incluido como variable de estudio la formación específica que estos profesionales tienen en riesgos ergonómicos, aspecto que apenas se estudia en otras investigaciones y que tiene una relevancia muy significativa en el ámbito preventivo.

\section{5. ¿Se han encontrado con alguna dificultad o contratiempo para el desarrollo del estudio?}

El principal contratiempo era conseguir una muestra suficientemente amplia para que las conclusiones tuvieran suficiente peso para ser consideradas relevantes y poder aumentar la sensibilización frente a los problemas que tienen estos profesionales. Afortunadamente, gracias a la colaboración de la Asociación Española de Técnicos de Laboratorio, AETEL, pudimos lograr una muestra bastante amplia que deja bien claro que es un colectivo muy afectado por dolencias musculares y que necesita que se trate de forma específica y singular frente al resto de sectores.

\section{6. ¿Se han obtenido los resultados esperados o se ha producido alguna sorpre- sa?}

De forma general hemos obtenido los resultados que esperábamos, un elevadísimo porcentaje de técnicos sufren molestias, siendo el cuello la parte más afectada. No obstante, aunque teníamos claro que la falta de formación era una variable que influía en gran medida, nos ha sorprendido la capacidad de amortiguación del riesgo que presenta. 
Aquellos técnicos que consideran tener formación específica en riesgos ergonómicos, reducen a la mitad la probabilidad de padecer dolencias musculoesqueléticas. El obtener datos cuantitativos de la relación existente entre ambos aspectos, hace mucho más palpable la importancia de establecer planes de formación específicos en este sector de actividad.

\section{7. ¿Cómo ha sido el proceso de publicación? ¿Han sufrido alguna incidencia?}

El proceso de publicación ha sido más que nada, largo y tedioso. Nuestro objetivo era publicar en una revista de cierto impacto para que el estudio tuviera la mayor difusión posible, así que apuntábamos a un JCR. Las normas y procesos de publicación de cada revista cambian, lo que implica que en cada envío debes reestructurar el artículo para cumplir todos los requisitos de cada una. A esto añadimos los tiempos de espera de respuesta de cada revista, las correcciones sugeridas por los árbitros, el tiempo entre la aceptación y la publicación, y nos vamos ya a tiempos que rondan el año.

Desde aquí nos gustaría hacer un llamamiento para que las revistas unificaran criterios en la medida de lo posible, para facilitar un poco este proceso.

\section{8. ¿Qué implicaciones tiene este estudio para la prevención de riesgos labora- les? ¿Cuál sería la recomendación para mejorar la práctica profesional en rela- ción al problema estudiado?}

Poner de manifiesto la problemática de los técnicos de laboratorio desde un punto de vista ergonómico y sensibilizar sobre la necesidad de realizar más estudios específicos para este sector, que aporten soluciones técnicas de mejora teniendo en cuenta que es una profesión feminizada.

Se requieren medidas de intervención en el entorno de trabajo, bajo criterios ergonómicos, como los establecidos en la NTP 1029 sobre requisitos de diseño, y se hace imprescindible implementar planes de formación de riesgos específicos, acordes a las actividades desarrolladas por estos profesionales.

\section{Resumen del artículo(1)}

Objetivo: analizar la prevalencia de síntomas musculoesqueléticos de origen laboral en los técnicos de laboratorio y su relación con los factores personales y organizacionales, así como con la falta de formación específica en riesgos ergonómicos.

Metodología: se aplica a una muestra de 460 técnicos de laboratorio, el Cuestionario Nórdico Estandarizado de Kuorinka validado en población española y una encuesta de caracterización de la muestra que contempla tanto variables personales como aspectos organizativo. Para realizar el análisis estadístico se aplicó el programa R.

Resultados: el $84.5 \%$ de la muestra estudiada presenta dolencias musculares, con mayor porcentaje en mujeres, siendo el cuello la parte más afectada. La probabilidad de sufrir molestias se multiplica por ocho en personas mayores de 46 años y las variables que se asociaron significativamente fueron: el sexo, el nivel de educación, la formación específica en riesgos ergonómicos y la antigüedad en el puesto de trabajo. 
Las principales causas asociadas fueron: posturas forzadas y movimientos repetitivos debido a tareas realizadas en campana, al deficiente diseño de alturas de planos de trabajo, al uso de micropipeta, microscopio, microtomo y ordenador.

Conclusiones: existe un elevado riesgo de padecer problemas músculoesqueléticos relacionados con la actividad de técnico de laboratorio. Se requieren medidas de intervención en el entorno de trabajo bajo criterios ergonómicos y se hace imprescindible implementar planes de formación de riesgos específicos, acordes a las actividades desarrolladas por estos profesionales.

\section{Referencias}

1. López-González MJ, González S, González-Menéndez E. Prevalence of musculoskeletal problems in laboratory technicians. International Journal of Occupational Safety and Ergonomics. 2019:1-12. doi:10.1080/10803548.2019.1646531. 